\title{
Dynamic contrast-enhanced MRI differentiates hepatocellular carcinoma from hepatic metastasis of rectal cancer by extracting pharmacokinetic parameters and radiomic features
}

\author{
JIANZHI LI ${ }^{1,2}$, FENG XUE ${ }^{1}$, XINGHUA XU ${ }^{1}$, QING WANG ${ }^{1}$ and XUEXI ZHANG ${ }^{3}$ \\ ${ }^{1}$ Department of Radiology, Qilu Hospital, Cheeloo College of Medicine, Shandong University, Jinan, \\ Shandong 250012; ${ }^{2}$ Department of Radiology, Jinan Infectious Disease Hospital, Cheeloo College of Medicine, \\ Shandong University, Jinan, Shandong $250021 ;^{3}$ GE Healthcare, Shanghai 200000, P.R. China
}

Received March 7, 2020; Accepted June 24, 2020

DOI: 10.3892/etm.2020.9115

\begin{abstract}
The aim of the present study was to explore how dynamic contrast-enhanced magnetic resonance imaging (DCE-MRI) may differentiate hepatocellular carcinoma (HCC) from hepatic metastasis of rectal cancer (HMRC) by extracting pharmacokinetic parameters and radiomic features. A total of 75 patients, including 41 cases with HCC and 34 cases with HMRC, underwent DCE-MRI examination. Dual-input two-compartment extended Tofts tracer kinetic model attached to a specialized image post-processing software package from OmniKinetics; GE Healthcare was used to calculate the values of the pharmacokinetic parameters and radiomic features, which were extracted from the lesions at the same region of interest. These values were evaluated using Student's t-test and receiver operating characteristic curves, and discriminant models were built to differentiate between HCC and HRMC. The results identified statistically significant differences in the values of the pharmacokinetic parameters hepatic perfusion index (HPI), endothelial transfer constant $\left(\mathrm{K}^{\text {trans }}\right)$, initial area under the gadolinium concentration curve during the first $60 \mathrm{sec}$ (IAUC) between the HCC and HRMC groups. In addition, statistically significant differences in 17 radiomic features were observed between the two groups $(\mathrm{P}<0.05)$. The
\end{abstract}

Correspondence to: Professor Qing Wang, Department of Radiology, Qilu Hospital, Cheeloo College of Medicine, Shandong University, 107 Wenhua West Road, Jinan, Shandong 250012, P.R. China

E-mail: wangqing663@163.com

Abbreviations: HCC, hepatocellular carcinoma; HMRC, hepatic metastasis of rectal cancer; DCE-MRI, dynamic contrast-enhanced magnetic resonance imaging; FDA, Fisher's discriminant analysis; IAUC, initial area under the gadolinium concentration curve during the first $60 \mathrm{sec}$; HPI, hepatic perfusion index

Key words: dynamic contrast-enhanced MRI, pharmacokinetics, radiomics, textural features, hepatocellular carcinoma, hepatic metastasis areas under the receiver operating characteristic (ROC) curves of the pharmacokinetic parameters $\mathrm{K}^{\text {trans }}$, IAUC and HPI were $0.73,0.77$ and 0.67 , respectively. The range of the areas under the ROC curves of the 17 radiomic features with statistical differences was 0.63-0.79. In addition, when pharmacokinetic parameters and radiomic features were incorporated, the area under the ROC curve was 0.86 . The accuracy of Fisher's discriminant analysis model based on radiomic features was $89.3 \%$, and the leave-one-out cross-validation accuracy was $80.0 \%$. In conclusion, DCE-MRI was demonstrated to be useful in the differential diagnosis of HCC and HMRC by extracting pharmacokinetic parameters and radiomic features, and incorporation of the two paths improved the diagnostic efficacy. A discriminant model based on radiomic features further enhanced the identification of HCC and HMRC.

\section{Introduction}

Hepatocellular carcinoma (HCC) and hepatic metastasis of rectal cancer (HMRC) are common malignant lesions of the liver, as well as major causes of mortality due to their high risk and rapid development (1-3). According to recent statistics, $\mathrm{HCC}$ is the fourth most common cause of cancer-associated mortality worldwide (4). The incidence of HMRC has been reported to be $\sim 25 \%$ at the time of diagnosis of rectal cancer (5). As these diseases exhibit distinctive biological activities, clinicians must identify the properties and tumor types of these hepatic lesions and provide different clinical treatment regimens to improve the surgical methods and patient survival rates.

Clinically, aside from laboratory examination, imaging-based differential diagnosis of these two diseases relies on traditional computed tomography (CT), magnetic resonance imaging (MRI) and ultrasonography (6). MRI was initially popular for the diagnosis of liver tumors; however, dynamic contrast-enhanced MRI (DCE-MRI) with quantitative or semi-quantitative functions has been gradually applied in clinical practice, as it provides a more comprehensive assessment of microvascular parameters and information in tumors compared with conventional contrast-enhanced MRI (7). Quantitative and semi-quantitative pharmacokinetic 
parameters can be produced by simulating the metabolic process of the contrast agent in the lesion (7). The endothelial transfer constant $\left(\mathrm{K}^{\text {trans }}\right)$ reflects the microvascular changes in the lesion area, and the $\mathrm{K}^{\text {trans }}$ value in the tumor area is markedly increased compared with that in normal tissue, which may be used to observe the vascular permeability changes of the liver and tumor tissues (8). The initial area under the gadolinium concentration curve during the first $60 \mathrm{sec}$ (IAUC) is a semi-quantitative parameter that represents the contrast agent concentration in the lesion during the first $60 \mathrm{sec}(9)$. Hepatic perfusion index (HPI) refers to the hepatic artery blood supply fraction related to the portal vein as a reference standard; lesions with different pathogeneses have different HPI values (10). Previous studies have demonstrated that quantified pharmacokinetic parameters, such as $\mathrm{K}^{\text {trans }}$, IAUC and HPI, derived from DCE-MRI exhibit high potential in assessing liver tumors (7-10).

Radiomics analysis is a high-throughput automated computing method used to transform the gray information of the region of interest (ROI) into high-dimensional image features in medical images (11). The image features may provide assistance and support for the diagnosis, treatment assessment and prognosis in clinical practice with precise quantitative analysis (11). Previous studies have reported the application of this analysis in the differential diagnosis and grade malignancy detection of non-small cell lung cancer, keratoma and prostate cancer (11). Compared with traditional technical methods, radiomics analysis provides more diagnostic and differential diagnosis information. For example, Huang et al (12) evaluated lymph node metastasis in patients with colorectal cancer by combining the lymph node status reported by $\mathrm{CT}$ and radiomics analysis, which may be conveniently used to facilitate preoperative individualized prediction. In another study, Huang et al (13) evaluated a subset of radiomic features extracted from CT images; in contrast with previous studies, CT-based radiomics analysis revealed its potential use as a predicted imaging biomarker in the diagnosis of non-small cell lung cancer. Radiomics analysis provides information that cannot be observed by the naked eye, and indicates the property of the lesions by evaluating the radiomic features extracted from them (11-13).

Differential diagnosis of HCC and HMRC would benefit patients and clinical practice. Therefore, the present study aimed to evaluate the ability of pharmacokinetic parameters, as well as radiomic features derived from DCE-MRI, to differentiate HCC from HMRC.

\section{Materials and methods}

Patient data. The patients provided written informed consent before undergoing DCE-MRI. A total of 75 patients (64 male and 11 female) with a mean age of $54.8 \pm 11.6$ years (range, 25-78 years) were consecutively recruited between December 2018 and July 2019. The recruited patients were divided into two groups according to histopathological results: i) HCC, $\mathrm{n}=41$ (35 male and 6 female); and ii) HMRC, $\mathrm{n}=34$ (25 male and 9 female). The patients were selected according to the following criteria (14): i) The HCC group lesions were obtained from patients who suffered from a single lesion; ii) the HRMC group included the largest lesion selected from patients with $\leq 3$ lesions; iii) the diameter of the lesions was $1-5 \mathrm{~cm}$ in both groups; iv) no contrast-enhanced MR examination had been performed within 30 days; v) all patients could follow the MR technician's request to complete the full scan. All lesions were histopathologically confirmed by surgery or biopsy 1 week after the MR examination. Patients with severe motion artifacts in the MRI and those who had received any antineoplastic treatment prior to their MR examination were excluded from the study.

MRI acquisition. Imaging of the whole liver was performed on a 3.0T HDX TwinSP MR system (GE Healthcare) using an 8-channel abdominal phased array body coil. Prior to the examination, breathing exercise training was provided to patients. Routine axial images, including T1-weighted images (TR/TE, 2.7/1.2 msec; FOV, 410x287 mm; slice thickness/space, 6/2 mm; slab, 24), T2-weighted images (TR/TE, 6670/87 msec; FOV, 410×287 mm; slice thickness/space, $6 / 2 \mathrm{~mm}$; slab, 24) and diffusion-weighted images (TR/TE, $5700 / 67 \mathrm{msec}$; b value, $1,000 \mathrm{sec} / \mathrm{mm}^{2}$; b value, 0 ), were first obtained. Multiple flip-angle images were collected using a 3D LAVA sequence (TR/TE, 2.8/1.3 msec; matrix, 288x188; FOV, 400x320 mm; phase FOV, 0.85; slice thickness/space, 6/2 mm; acceleration, 2.50; slab, 64; time resolution, $6.0 \mathrm{sec}$ ) with in-flip angles of $3,6,9,12$ and $15^{\circ}$ (14).

DCE-MRI was performed with 22 acquiring phases in a 12-flip angle (14). Two unenhanced phases were first collected as the baseline. A contrast agent (Omniscan; GE Healthcare) was injected into the elbow vein at $2.0 \mathrm{ml} / \mathrm{sec}$ $(0.3 \mathrm{mmol} / \mathrm{kg})$ using a high-pressure injector (Mississippi ${ }^{\mathrm{TM}}$ XD 2000 Injector; Ulrich GmbH \& Co. KG) and was effective from the third phase. Following injection, $20 \mathrm{ml}$ saline was delivered at a rate of $2.0 \mathrm{ml} / \mathrm{sec}$ to flush the injector and its accessory tube.

Data acquisition. A specialized medical image post-processing software package (OmniKinetics V2.0.10, GE Healthcare) for DCE-MRI was used in the present study $(14,15)$. First, the multiple flip angle images $\left(3,6,9,12\right.$ and $\left.15^{\circ}\right)$ were imported into the software for T1 mapping calculation, and all DCE-MR images aligned by $3 \mathrm{D}$ non-rigid registration function, which attached to the OmniKinetics software, were subsequently loaded. Secondly, a dual-input two-compartment tracer kinetic liver model termed Extended Tofts was selected from OmniKinetics. Hepatic artery and portal vein ROIs were drawn by hand, and were performed by two radiologists in consensus, both with 8 and 12 years of experience, respectively, to obtain the arterial input function (AIF) and portal input function (PIF) of the contrast agent time-concentration curve. The contrast agent time-concentration curve of the dual vascular input function (VIF) in the liver (Fig. 1) was fitted by AIF and PIF. The ROI of the hepatic artery was placed on the abdominal aorta near the entrance of the celiac trunk, replacing the hepatic artery, and the ROI of the portal vein was placed on the main portal vein. Thirdly, two radiologists with 8 and 12 years of experience in MRI reviewed the DCE-MRI, and the images were selected from the phase that corresponded to the peak of hepatic artery enhancement based on AIF. A consensus was reached through consultation. An oval or polygonal ROI was manually drawn along the edge of the largest cross-section 

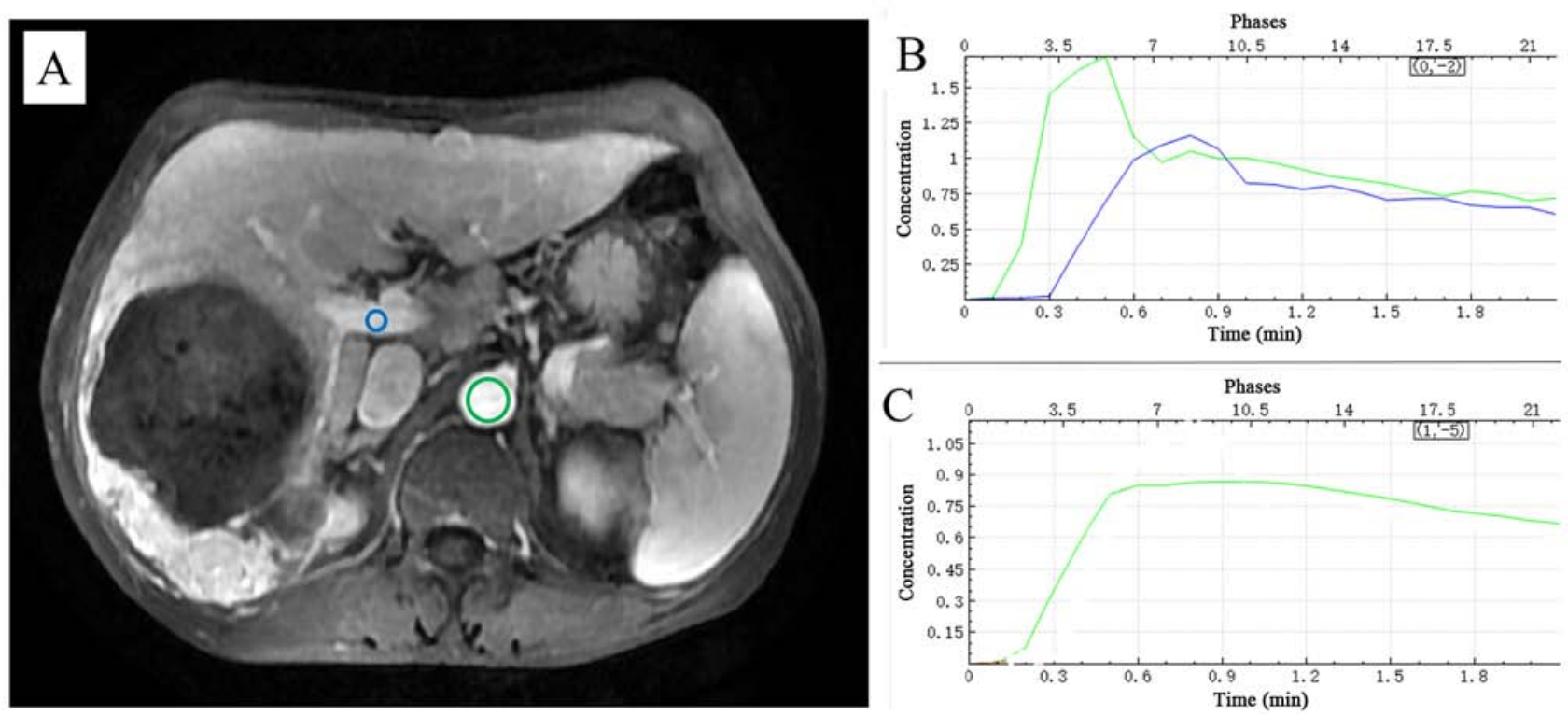

Figure 1. Acquisition process of the dual VIF. (A) The ROI of the hepatic artery was placed on the abdominal aorta near the entrance of the celiac trunk, as indicated by the green circle. The ROI of the portal the vein was placed on the main portal vein, as indicated by the blue circle. The area of each centered ROI was approximately two-thirds of its vascular cross-section. (B) AIF is indicated by the green curve, and PIF is indicated by the blue curve. (C) The contrast agent time-concentration curve of the dual VIF was fitted by AIF and PIF. VIF, vascular input function; ROI, region of interest; AIF, arterial input function; PIF, portal input function.
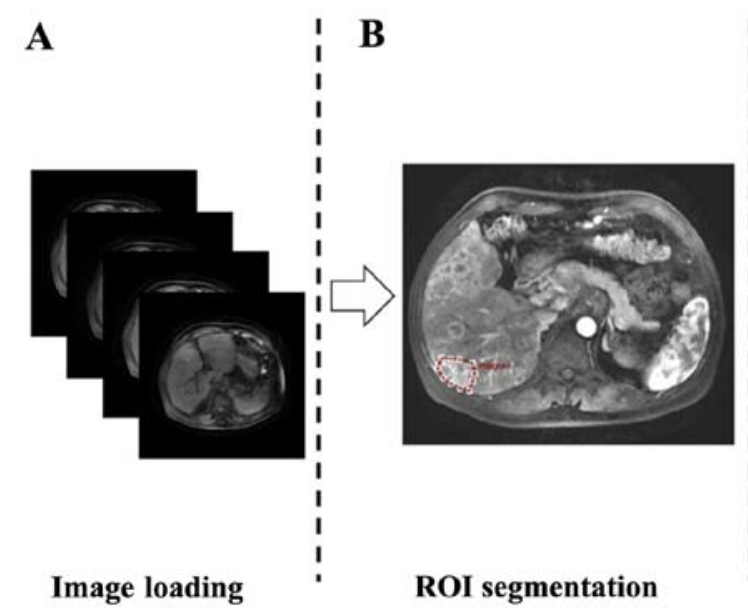
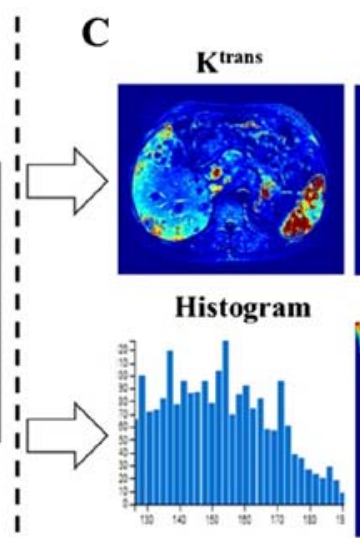

Pharmacokinetic and radiomic parameters

Figure 2. Acquisition of pharmacokinetic parameters and radiomic features derived from DCE-MRI. (A) Loading images in dicom format. (B) Manually drawn ROI of the lesion; image segmentation was obtained. (C) Calculation of pharmacokinetic parameters and radiomic features. DCE-MRI, dynamic contrast-enhanced magnetic resonance imaging; ROI, region of interest; $\mathrm{K}^{\text {trans }}$, endothelial transfer constant; IAUC, initial area under the gadolinium concentration curve during the first $60 \mathrm{sec}$; HPI, hepatic perfusion index; GLCM, gray level co-occurrence matrix; RLM, run length matrix.

of the lesion, and image segmentation of the ROI lesion was obtained. Finally, pharmacokinetic parameters and radiomic features were calculated using the 'calculate' function of the OmniKinetic software. The values of the pharmacokinetic parameters were obtained, including $\mathrm{K}^{\text {trans }}$, IAUC and HPI, which contributed to the differentiation of liver tumors in previous studies (7-10) and were thus used in the present study. The values of radiomic features were calculated using the 'CalcTexturePragram' function of the software (Fig. 2). Three successive ROIs based on the largest cross-section of the lesion, including the upper and lower layers, were measured for pharmacokinetic parameters and radiomic features, and the mean values of the three measurements were calculated by clicking the 'merge' button on the software.
The radiomic features derived from the lesion DCE-MRI were selected in the phase when AIF reached the peak of the contrast agent time-concentration curve of the hepatic artery $(14,15)$. These parameters, which were calculated by OmniKinetics software, included five types of features: First order, histogram, gray level co-occurrence matrix, Haralick and run length matrix. The features are listed in Table I.

Statistical analysis. R package version 3.5.0 (https://www. Rproject.org) was used for the present study (16). Normally distributed continuous variables, including the values of pharmacokinetic parameters and radiomic features, are presented as the mean \pm standard deviation. Student's t-test was used to compare these variables between the HCC and 
Table I. Radiomic features derived from dynamic contrast-enhanced magnetic resonance imaging by OmniKinetics software.

\begin{tabular}{|c|c|c|}
\hline Type & Radiomic features & $\mathrm{N}$ \\
\hline First order & $\begin{array}{l}\text { MinIntensity, MaxIntensity, MedianIntensity, MeanValue, stdDeviation, Variance, } \\
\text { VolumeCount, VoxelValueSum, Root Meant Square, Range, MeanDeviation, } \\
\text { RelativeDeviation, MinLocation, MaxLocation }\end{array}$ & 14 \\
\hline Histogram & $\begin{array}{l}\text { Energy, Entropy, Kurtosis, Skewness, Uniformity, FrequencySize, Uniformity Positive } \\
\text { Pixel, Mean Positive Pixel, Quantile5, Quantile10, Quantile25, Quantile50, Quantile75, } \\
\text { Quantile90, Quantile95 }\end{array}$ & 15 \\
\hline GLCM & $\begin{array}{l}\text { GlcmEnergy, GlcmEntropy, GlcmBinSize, GlcmTotalFrequency, GlcmMatrixMean, } \\
\text { GlcmRelativeFrequency, Inertia, Correlation, InverseDifferenceMoment, ClusterShade, } \\
\text { ClusterProminence, HaralickCorrelation, InvalidFeatureName }\end{array}$ & 13 \\
\hline Haralick & $\begin{array}{l}\text { AngularSecondMoment, Contrast, Hara Variance, sumAverage, sumVariance, sumEntropy, } \\
\text { differenceVariance, differenceEntropy, inverseDifferenceMoment }\end{array}$ & 9 \\
\hline RLM & $\begin{array}{l}\text { MaxIntensity, MinIntensity, MinSize, NumberOfIntensityBins, MaxSize, NumberOfSizeBins, } \\
\text { ShortRunEmphasis, LongRunEmphasis, GreyLevelNonuniformity, RunLengthNonuniformity, } \\
\text { LowGreyLevelRunEmphasis, HighGreyLevelRunEmphasis, ShortRunLowGreyLevelEmphasis, } \\
\text { ShortRunHighGreyLevelEmphasis, LongRunLowGreyLevelEmphasis, } \\
\text { LongRunHighGreyLevelEmphasis }\end{array}$ & 16 \\
\hline
\end{tabular}

GLCM, gray level co-occurrence matrix; RLM, run length matrix.

Table II. Clinicopathological characteristics of patients with HCC and HMRC.

\begin{tabular}{|c|c|c|c|c|}
\hline Characteristic & $\operatorname{HCC}(n=41)$ & HMRC (n=34) & $\mathrm{t} / \chi^{2}$ & P-value \\
\hline Age, years & $51.59 \pm 10.25$ & $53.88 \pm 13.21$ & 0.61 & 0.54 \\
\hline \multicolumn{5}{|l|}{ Sex, n $(\%)$} \\
\hline Male & $35(85.37)$ & $29(85.29)$ & $<0.01$ & $>0.99$ \\
\hline Female & $6(14.63)$ & $5(14.71)$ & & \\
\hline
\end{tabular}

HCC, hepatocellular carcinoma; HMRC, hepatic metastasis of rectal cancer.

HMRC groups. The sensitivity, specificity, cut-off value, area under the curve (AUC) and 95\% confidence interval (CI) were calculated by receiver operating characteristic (ROC) curve using the 'pROC' package (17). Binary logistic regression was used to analyze the variables to differentiate between HCC and HMRC using pharmacokinetic parameters and radiomic features with statistical differences as independent variables, and pathological results as dependent variables. A combination of variables was produced after eliminating the variables. Fisher discriminant analysis (FDA) and leave-one-out cross-validation were used to build linear discriminant models. Two-tailed $\mathrm{P}<0.05$ was considered to indicate a statistically significant difference.

\section{Results}

Patients. The age range of the 35 male and 6 female patients in the HCC group was $25-75$ years. The age range of 29 male and 5 female patients in the HMRC group was 26-78 years. No significant differences were observed in the age and sex between the two groups (Table II; P>0.05).
Pharmacokinetic parameters and radiomic features. Statistically significant differences in $\mathrm{K}^{\text {trans }}$, IAUC and HPI values were observed between the HCC and HRMC groups (Table III). In addition, statistically significant differences were identified in 17 features between the two groups (Table IV).

Efficacy of pharmacokinetic parameters and radiomic features. The areas under the ROC curves of pharmacokinetic parameters $\mathrm{K}^{\text {trans }}$, IAUC and HPI were $0.73(0.61-0.84,95 \%$ CI), $0.77(0.67-0.88,95 \% \mathrm{CI})$ and $0.67(0.55-0.79,95 \% \mathrm{CI})$, respectively (Table III and Fig. 3). The areas under the ROC curves of the 17 radiomic features with statistical differences were between 0.63 and 0.79 (Table IV and Fig. 4). The combination of pharmacokinetic parameters and radiomic features was termed the P-R parameter. The area under the ROC curve of the P-R parameter was 0.86 (95\% CI, 0.77-0.94), with a sensitivity of $90.24 \%$ and specificity of $73.53 \%$ (Fig. 5).

Discriminant models based on the radiomic features. The training dataset comprised the 67 radiomic features from the two groups (a total of 75 cases). Accordingly, 14 radiomic 


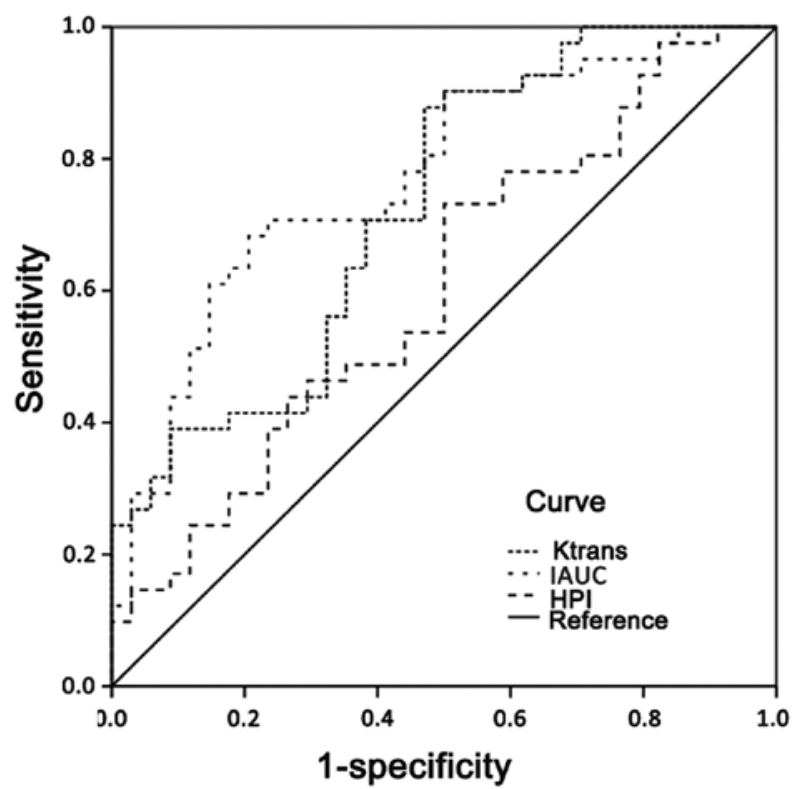

Figure 3. Receiver operating characteristic curves of the pharmacokinetic parameters. $\mathrm{K}^{\text {trans }}$, endothelial transfer constant; IAUC, initial area under the gadolinium concentration curve during the first $60 \mathrm{sec}$; HPI, hepatic perfusion index.

features were enrolled to build the discriminant functions required to differentiate HCC from HMRC by calculating the correlation coefficient of intraclass and interclass cases as follows:

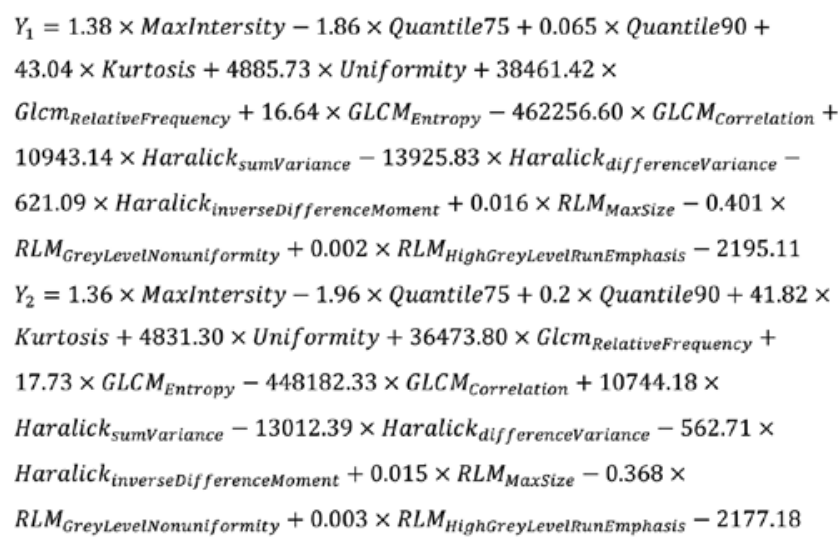

Where $\mathrm{Y}_{\mathrm{i}}\left(\mathrm{Y}_{1}, \mathrm{HCC} ; \mathrm{Y}_{2}, \mathrm{HMRC}\right)$ refers to the corresponding scores that determine the tumor classification. If the score of one specified lesion was close to 1 , the corresponding case was recognized as HCC. If the score of one specified lesion was close to 2, the case was recognized as HMRC. The FDA model automatically iterated 67 times to achieve accuracy, and the leave-one-out cross-validation method was performed to validate that accuracy (Table V).

\section{Discussion}

In the present retrospective study, in order to evaluate HCC and HMRC in the DCE-MRI of lesions, two types of parameters were examined, namely pharmacokinetic parameters and radiomic features, and their values were comprehensively analyzed. Both types of parameters contributed to differentiating $\mathrm{HCC}$ from HRMC, and their incorporation improved the 


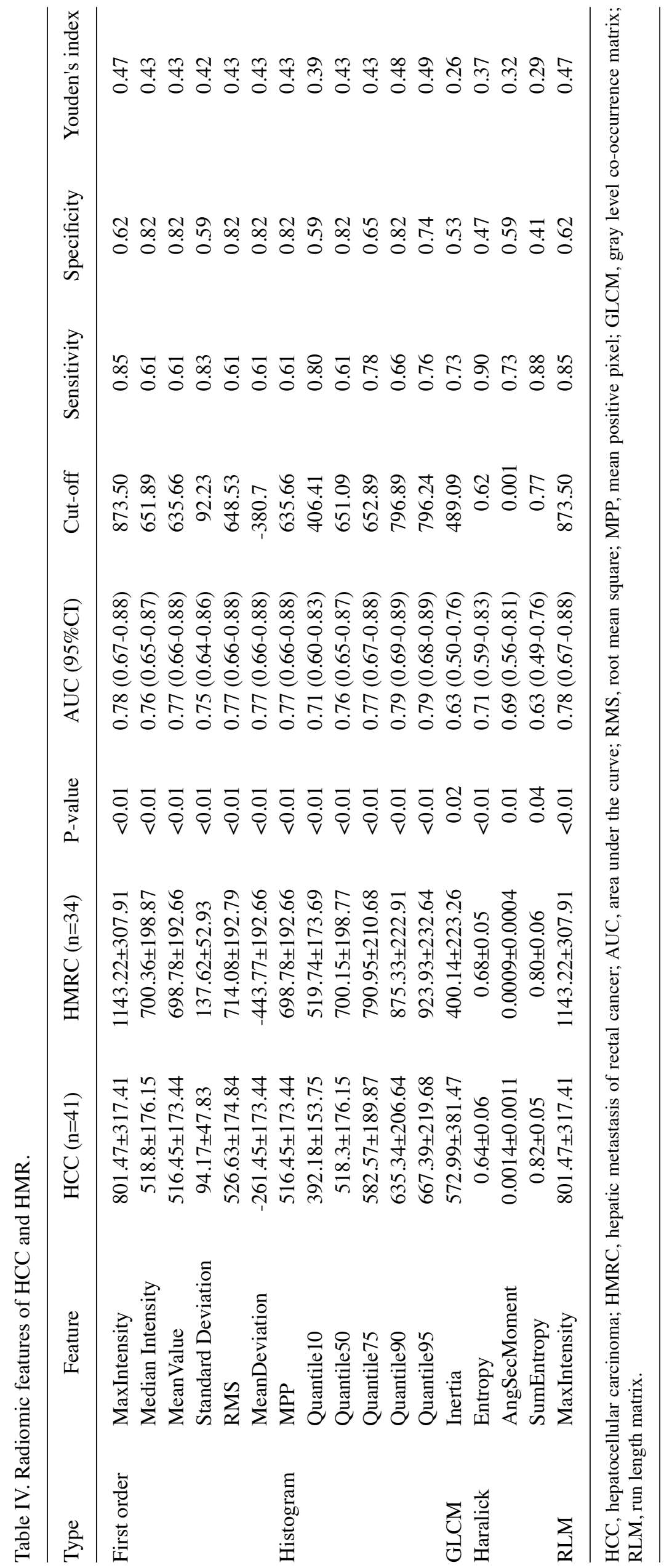



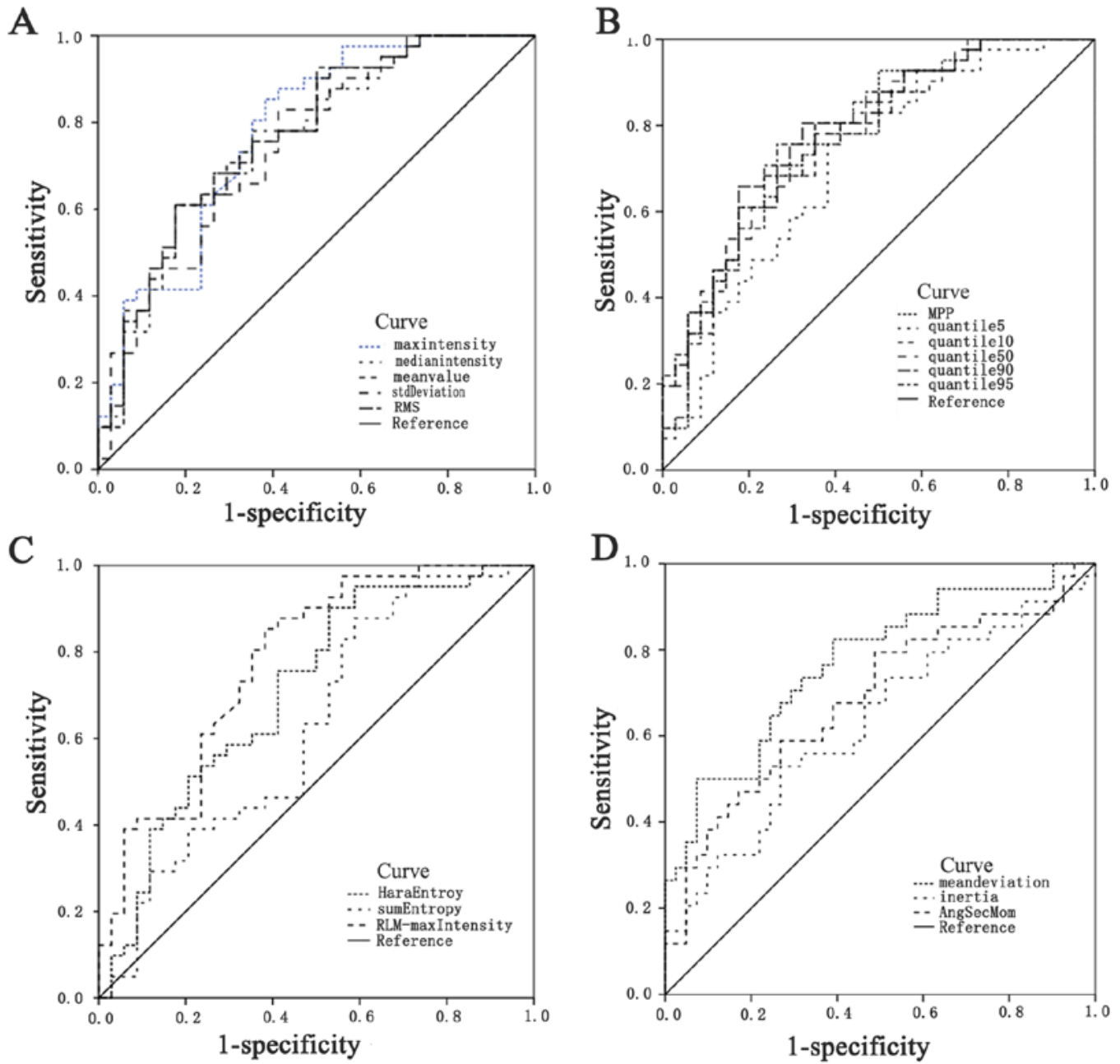

Figure 4. Receiver operating characteristic curves of the radiomic features. (A) Max intensity,Median intensity,Mean value,Standard deviation,RMS; (B) MPP, Quantile5,Quantile10, Quantile50,Quantile90,Quantile95; (C) Haralick entropy,Sum entropy, RLM-Max intensity; (D) Mean deviation,Inertia, AngSecMoment. RMS,root mean square; MPP,mean positive pixel; RLM,run length matrix; AngSecMom,angular second moment.

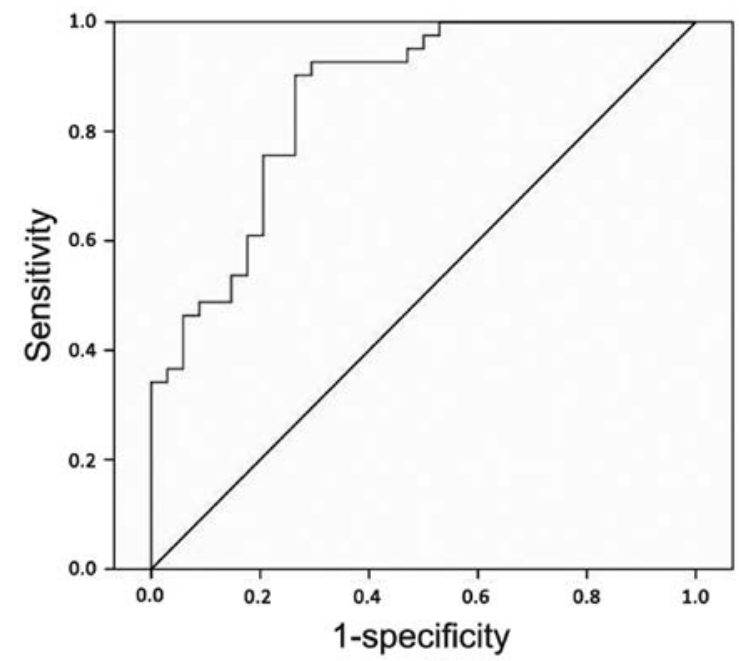

Figure 5. Receiver operating characteristic curve of the P-R parameter. $\mathrm{P}-\mathrm{R}$, pharmacokinetic parameters and radiomic features.

efficacy of diagnosis compared with that of either type alone. In addition, the discriminant model based on the radiomic features further enhanced the identification of HCC and HMRC.
In the present study, a number of measures were taken to acquire accurate data (18-21). First, the same MR scanner was used for all patients. Scanning was performed at the end-expiratory breath holding, and 3D non-rigid registration was used to reduce motion artifacts. Contrast agent was simultaneously injected and scanned, and the scanning interval was $\leq 6 \mathrm{sec}$ in the four following phases to ensure that the images with the most notable enhancement phase of the hepatic artery were captured. A total of 22 phases were scanned to uniformly obtain successive and complete DCE-MRIs. Following an elbow vein injection in all cases, the constants of the injection rate and concentration were determined to ensure the consistency of the contrast agent concentration perfusion to the liver in correspondence with VIF. These measures also prevented any differences caused by various contrast agent concentrations. Secondly, an extended Tofts with dual-input two-compartment model was selected instead of the traditional two-compartment Tofts model. The selected model considered hepatic artery and portal vein input to the liver. Images of the derived radiomic features were selected from the phase when AIF reached the peak; images from this phase were the most representative of disease characteristics and revealed the most information about these characteristics. Thirdly, in image 
Table V. Results of the discriminant models based on the radiomic features.

\begin{tabular}{lllrrr}
\hline \multirow{2}{*}{ Method } & & & \multicolumn{2}{c}{ Prediction } & \\
FDA training dataset & Group & $\mathrm{N}$ & HCC $(\%)$ & HMRC (\%) & Discriminant accuracy (\%) \\
Leave-one-out cross-validation & HCC & 41 & $39(95.1)$ & $2(4.9)$ & \multirow{2}{*}{89.3} \\
& HMRC & 34 & $6(17.6)$ & $28(82.4)$ & \\
& HCC & 41 & $35(85.4)$ & $6(14.6)$ & \multirow{2}{*}{80.0} \\
& HMRC & 34 & $9(26.5)$ & $25(73.5)$ & \\
\hline
\end{tabular}

HCC, hepatocellular carcinoma; HMRC, hepatic metastasis of rectal cancer; FDA, Fisher's discriminant analysis.

segmentation, vascular, cystic and necrotic areas were avoided by manually drawing the ROIs to ensure the precision of the selected area. Three successive layers were measured to avoid statistical accidental error in the selected lesions. All measures taken in this experiment were conducive to obtaining the accurate values of pharmacokinetic parameters and radiomic features.

As a non-invasive and radiation-free examination method, MRI reveals the internal information of malignant tumors from multiple perspectives using different imaging technology modes. In addition, DCE-MRIs were also indicated in addition to the enhancement characteristics. The perfusion and permeability parameters obtained though the pharmacokinetic model may be used to assess the changes microvascular blood supply to the tumor (22). These functional pharmacokinetic parameters, such as $\mathrm{K}^{\text {trans }}$, IAUC and HPI, may reflect the hemodynamic changes of microvessels in the tumor region, describe the characteristics of tumor lesions and reflect the properties of the lesions from different perspectives to identify tumors to a certain extent $(21,23) . \mathrm{K}^{\text {trans }}$ refers to the rate by which the contrast agent leaks into the extracellular fluid space outside blood vessels and is associated with total blood perfusion, vascular surface area and vascular permeability, thus reflecting the changes in the integrity of tissue microvessels (8). Compared with normal tissues, tumor tissues are characterized by an abundance of nascent capillaries, higher microvascular density, larger vascular osmotic surface area, immature microvessels, wider endothelial cell space, incomplete basement membrane and pathological basement membrane structure; this results in increases in tumor tissue microvascular permeability and its $\mathrm{K}^{\text {trans }}$ value (24). In the present study, the $\mathrm{K}^{\text {trans }}$ value of HCC was higher compared with that of HRMC, indicating that HCC exhibited a higher level of infiltration and permeability compared with those of HRMC on the vascular surface, which may be used for the differential diagnosis of the two lesions. The IAUC value, which was represented by the AUC of the time-concentration curve of the contrast agent, refers to the estimated value of the concentration of the contrast agent in the lesion (9). The degree of enhancement varies for lesions with different etiologies, and the IAUC value may be used to identify lesions with different properties $(19,21)$. The results of the present study demonstrated that the IAUC values of the two groups were significantly different, suggesting that IAUC discriminated between the two lesions, which was consistent with a previously published study (25). The perfusion parameter HPI is the perfusion ratio of the hepatic artery, and the hepatic artery provides $20-30 \%$ of the blood supply under the physiological status of the liver (26). The biological behavior of the tumor, which includes tumorigenesis, growth and progression, depends on angiogenesis; therefore, different types of tumors have different blood supplies (26). HCC is usually associated with a rich blood supply that mainly originates from the hepatic artery (26). However, the richness or poorness of the blood supply of the tumor remains controversial. Previous studies have demonstrated that the hepatic artery is the main source of blood supply for HMRC, regardless of whether the blood supply is rich or poor $(19,27)$. The results of the present study revealed that the HPI values of the two groups were significantly higher compared with those of normal liver tissue, confirming that the hepatic artery was the main source of blood supply for HCC and HMRC tumors. In addition, the HPI values between the two groups were significantly different. This result was consistent with the findings of previous studies, which demonstrated that HPI distinguished HCC from HMRC $(19,27)$. The pharmacokinetic parameters $\mathrm{K}^{\text {trans }}$, IAUC and HPI reflect the relevant characteristics and properties of lesions from different perspectives, which may be used to identify HCC and HMRC.

Radiomics analysis, which uses the law of change and distribution of image pixel gray value, is used as a mathematical engineering method to express radiomics characteristic information (28). The differences in medical images caused by tumor heterogeneity can be quantified using radiomic features. Therefore, radiomics data are potentially associated with pathology (29). In addition, radiomics data provide quantitative information about tumor characteristics and have the potential to discover disease characteristics that cannot be observed by the naked eye by combining mathematics, engineering and medical science (30). DCE-MRI based on anatomical structure provides functional information, including that on perfusion and metabolism, due to differences in lesion tissues (31). The functional information differences in DCE images of biological characteristics and heterogeneity of tumors may be reflected by the values of the radiomic features $(31,32)$. In addition, the present study confirmed that radiomics analysis could be used in the differential diagnosis of HCC and HMRC. In the present study, 67 radiomic features were obtained using OmniKinetic software; however, only 17 features exhibited significant differences, indicating that there 
were several common features in the tumor characteristics between HCC and HMRC lesions, including blood supply and microvascular changes. Identifying these images by the naked eye is challenging; however, after considerable information screening, highly efficient radiomic features of the identity of the two lesions were obtained. Therefore, radiomic feature analysis may help clinicians identify HCC and HMRC.

In the present study, ROC curves and logistic regression were used for analysis. ROC curves used AUC to evaluate the diagnostic efficiency of each pharmacokinetic parameter and radiomic feature. These high values of AUCs of $\mathrm{K}^{\text {trans }}$, IAUC, HPI and radiomic features indicate that pharmacokinetic parameters and radiomic features effectively differentiated HCC from HMRC (Tables III and IV; Figs. 3 and 4). The AUC of incorporated parameter P-R was 0.86 , which was higher compared with that of each of the pharmacokinetic parameters and radiomic features alone. P-R was also demonstrated to have high sensitivity and specificity. Therefore, it can be concluded that combining pharmacokinetics and high-accuracy radiomics improved the detection rate of HCC.

Radiomic features and regression model analysis for evaluating disease diagnosis, development, treatment and prognosis have become popular in recent years (33). In the present study, the mathematical model FDA was applied for the analysis of the radiomic features. FDA established the discriminant model to determine the minimum covariance between samples within the same category, and maximum covariance between samples within different categories. The FDA method provided two discriminant functions $\mathrm{Y}_{1}$ and $\mathrm{Y}_{2}$ for $\mathrm{HCC}$ and HMRC, respectively. The category of unidentified liver tumors may be easily recognized through this model, which can automatically draw conclusions using statistical calculations. The FDA model exhibited $89.3 \%$ discriminant accuracy and good calibration with $80 \%$ accuracy within the datasets of the two groups, thereby showing marked discrimination. To the best of our knowledge, previous studies propounded several radiomics methods for the differentiation of tumors, but a limited number of studies have provided discriminant functions (34). Compared with complicated discriminant methods, FDA is an accessible approach that considers intergroup and intragroup covariance (33). However, the present study is a pilot study of differentiation research on HCC and HMRC; considering the limited number of cases, no additional patients were used as an external dataset to validate the regression. This will be addressed in a future study.

The present study had several limitations. The MRI system, sequences, time resolution, perfusion time, contrast agent, dose, concentration and injection rate affected the value of the parameters; therefore, the findings might differ from those of previous studies (35). However, the study design was based on the same reference standard for all patients. Secondly, the radiomic features extracted from DCE-MRI may be different from those presented in previous studies due to variations in radiomics analysis software (36). Thirdly, radiomics and FDA require further multicenter analysis with a large sample size.

In conclusion, the present study demonstrated that DCE-MRI was useful for the differential diagnosis of HCC and HMRC by extracting pharmacokinetic parameters and radiomic features, and the incorporation of the two methods improved the diagnostic efficacy. When mathematical engineering techniques are adequately exploited, the discriminant models based on the radiomic features have potential to diagnose unidentified liver tumors. Thus, this method is worthy of further exploration in clinical settings.

\section{Acknowledgements}

The authors would like to thank Professor Dechao Feng and Dr Meng Zhao (Department of Radiology, Qilu Hospital, Shandong University, Jinan, China), Dr Jieqiong Wang (Department of Radiology, Taikang Xianlin Drum Tower Hospital, Nanjing, China) and Dr Yumei Zhang (Department of Radiology, Yantai Yuhuangding Hospital, Qingdao Medical College, Yantai, China) for their advice on the operation of MRI technology. The current study was supported by Dr Xiao Xu (GE Healthcare, Shanghai, China) on the technology of OmniKinetic software application.

\section{Funding}

Not applicable.

\section{Availability of data and materials}

The datasets used and/or analyzed during the current study are available from the corresponding author on reasonable request.

\section{Authors' contributions}

ZJL contributed to the conception and design of the study, data acquisition and analysis, figure modification and writing of the manuscript. FX and XHX contributed to the data acquisition. QW contributed to the conception and design of the study and revising of the manuscript. XXZ contributed to the conception and design of the study, data analysis and figure creation. All authors read and approved the final manuscript.

\section{Ethics approval and consent to participate}

The present study was approved by Ethics Committee for Human Studies, Qilu Hospital, Cheeloo College of Medicine, Shandong University (Shandong, China), and written informed consent was obtained from all patients.

\section{Patient consent for publication}

Not applicable.

\section{Competing interests}

$\mathrm{XXZ}$ is affiliated with GE Healthcare, which provided computer equipment with the OmniKinetics software installed and technology support for this study. All other authors declare that they have no competing interests.

\section{References}

1. Bosetti C, Turati F and La Vecchia C: Hepatocellular carcinoma epidemiology. Best Pract Res Clin Gastroenterol 28: 753-770, 2014. 
2. Hartke J, Johnson M and Ghabril M: The diagnosis and treatment of hepatocellular carcinoma. Semin Diagn Pathol 34: 153-159, 2017.

3. Balyasnikova S and Brown G: Optimal imaging strategies for rectal cancer staging and ongoing management. Curr Treat Options Oncol 17: 32, 2016.

4. Yang JD, Hainaut P, Gores GJ, Amadou A, Plymoth A and Roberts LR: A global view of hepatocellular carcinoma: Trends, risk, prevention and management. Nat Rev Gastroenterol Hepatol 16: 589-604, 2019.

5. Kye BH, Lee SH, Jeong WK, Yu CS, Park IJ, Kim HR, Kim J, Lee IK, Park KJ, Choi HJ, et al: Which strategy is better for resectable synchronous liver metastasis from colorectal cancer, simultaneous surgery, or staged surgery? Multicenter retrospective analysis. Ann Surg Treat Res 97: 184-193, 2019.

6. Lamba R, Fananapazir G, Corwin MT and Khatri VP: Diagnostic imaging of hepatic lesions in adults. Surg Oncol Clin N Am 23: 789-820, 2014

7. Kim H, Mousa M, Schexnailder P, Hergenrother R, Bolding M, Ntsikoussalabongui B, Thomas V and Morgan DE: Portable perfusion phantom for quantitative DCE-MRI of the abdomen. Med Phys 44: 5198-5209, 2017.

8. Chen BB, Hsu CY, Yu CW, Liang PC, Hsu C, Hsu CH, Cheng AL and Shih TT: Early perfusion changes within 1 week of systemic treatment measured by dynamic contrast-enhanced MRI may predict survival in patients with advanced hepatocellular carcinoma. Eur Radiol 27: 3069-3079, 2017.

9. Chouhan MD, Bainbridge A, Atkinson D, Punwani S, Mookerjee RP, Lythgoe MF and Taylor SA: Improved hepatic arterial fraction estimation using cardiac output correction of arterial input functions for liver DCE MRI. Phys Med Biol 62: 1533-1546, 2017.

10. Joo I, Lee JM, Han JK, Yang HK, Lee HJ and Choi BI: Dynamic contrast-enhanced MRI of gastric cancer: Correlation of the perfusion parameters with pathological prognostic factors. J Magn Reson Imaging 41: 1608-1614, 2015.

11. Lambin P, Leijenaar RTH, Deist TM, Peerlings J, de Jong EEC, van Timmeren J, Sanduleanu S, Larue RTHM, Even AJG, Jochems A, et al: Radiomics: The bridge between medical imaging and personalized medicine. Nat Rev Clin Oncol 14 749-762, 2017.

12. Huang Y, Liang C, He L, Tian J, Liang CS, Chen X, Ma ZL and Liu ZY: Development and validation of a radiomics nomogram for preoperative prediction of lymph node metastasis in colorectal cancer. J Clin Oncol 34: 2157-2164, 2016.

13. Huang Y, Liu Z, He L, Chen X, Pan D, Ma Z, Liang C, Tian J and Liang C: Radiomics signature: A potential biomarker for the prediction of disease-free survival in early-stage (I or II) non-small cell lung cancer. Radiology 281: 947-957, 2016.

14. Yang JF, Zhao ZH, Zhang Y, Zhao L, Yang LM, Zhang MM, Wang BY, Wang T and Lu BC: Dual-input two-compartment pharmacokinetic model of dynamic contrast-enhanced magnetic resonance imaging in hepatocellular carcinoma. World J Gastroenterol 22: 3652-3662, 2016.

15. Tao X, Wang L, Hui Z, Liu L, Ye F, Song Y, Tang Y, Men Y, Lambrou T, Su Z, et al: DCE-MRI Perfusion and permeability parameters as predictors of tumor response to CCRT in patients with locally advanced NSCLC. Sci Rep 6: 35569, 2016.

16. R Core Team: R: A language and environment for statistical computing. R Foundation for Statistical Computing, Vienna, Austria, 2013.

17. Li J, Wang W, Xia P, Wan L, Zhang L, Yu L, Wang L, Chen X, $\mathrm{Xiao} Y$ and $\mathrm{Xu} C$ : Identification of a five-lncRNA signature for predicting the risk of tumor recurrence in patients with breast cancer. Int J Cancer 143: 2150-2160, 2018.

18. Heacock L, Gao Y, Heller SL, Melsaether AN, Babb JS, Block TK, Otazo R, Kim SG and Moy L: Comparison of conventional DCE-MRI and a novel golden-angle radial multicoil compressed sensing method for the evaluation of breast lesion conspicuity. J Magn Reson Imaging 45: 1746-1752, 2017.

19. Ginsburg SB, Algohary A, Pahwa S, Gulani V, Ponsky L, Aronen HJ, Boström PJ, Böhm M, Haynes AM, Brenner P, et al: Radiomic features for prostate cancer detection on MRI differ between the transition and peripheral zones: Preliminary findings from a multi-institutional study. J Magn Reson Imaging 46: 184-193, 2017.
20. Aronhime S, Calcagno C, Jajamovich GH, Dyvorne HA, Robson P, Dieterich D, Fiel MI, Martel-Laferriere V, Chatterji M, Rusinek H and Taouli B: DCE-MRI of the liver: Effect of linear and nonlinear conversions on hepatic perfusion quantification and reproducibility. J Magn Reson Imaging 40: 90-98, 2014.

21. Grossmann P, Narayan V, Chang K, Rahman R, Abrey L, Reardon DA, Schwartz LH, Wen PY, Alexander BM, Huang R and Aerts HJWL: Quantitative imaging biomarkers for risk stratification of patients with recurrent glioblastoma treated with bevacizumab. Neuro Oncol 19: 1688-1697, 2017.

22. O'Neill AF, Qin L, Wen PY, de Groot JF, Van den Abbeele AD and Yap JT: Demonstration of DCE-MRI as an early pharmacodynamic biomarker of response to VEGF Trap in glioblastoma. J Neurooncol 130: 495-503, 2016.

23. Lee SH, Hayano K, Zhu AX, Sahani DV and Yoshida H: Dynamic contrast-enhanced MRI kinetic parameters as prognostic biomarkers for prediction of survival of patient with advanced hepatocellular carcinoma: A pilot comparative study. Acad Radiol 22: 1344-1360, 2015.

24. Gandhi M, Choo SP, Thng CH, Tan SB, Low AS, Cheow PC, Goh AS, Tay KH, Lo RH, Goh BK, et al: Single administration of selective internal radiation therapy versus continuous treatment with sorafeNIB in locally advanced hepatocellular carcinoma (SIRveNIB): Study protocol for a phase III randomized controlled trial. BMC Cancer 16: 856, 2016.

25. Gaeta M, Benedetto C, Minutoli F, D'Angelo T, Amato E, Mazziotti S, Racchiusa S, Mormina E, Blandino A and Pergolizzi S: Use of diffusion-weighted, intravoxel incoherent motion, and dynamic contrast-enhanced MR imaging in the assessment of response to radiotherapy of lytic bone metastases from breast cancer. Acad Radiol 21: 1286-1293, 2014.

26. Şen H, Tan YZ, Binnetoğlu E, Aşik M, Güneş F, Erbağ G, Gazi E, Cevizci S, Özdemir S, Akbal E and Ükinc K: Evaluation of liver perfusion in diabetic patients using $99 \mathrm{mTc}$-sestamibi. Wien Klin Wochenschr 127: 19-23, 2015.

27. Kusano M, Honda M, Okabayashi K, Akimaru K, Kino S, Tsuji Y, Watanabe M, Suzuki S, Yoshikawa T, Sakamoto J, et al: Randomized controlled phase III study comparing hepatic arterial infusion with systemic chemotherapy after curative resection for liver metastasis of colorectal carcinoma: JFMC 29-0003. J Cancer Res Ther 13: 84-90, 2017.

28. Avanzo M, Stancanello J and El Naqa I: Beyond imaging: The promise of radiomics. Phys Med 38: 122-139, 2017.

29. Peeken JC, Nüsslin F and Combs SE: 'Radio-oncomics': The potential of radiomics in radiation oncology. Strahlenther Onkol 193: 767-779, 2017.

30. Peeken JC, Bernhofer M, Wiestler B, Goldberg T, Cremers D, Rost B, Wilkens JJ, Combs SE and Nüsslin F: Radiomics in radiooncology-challenging the medical physicist. Phys Med 48: 27-36, 2018.

31. Limkin EJ, Sun R, Dercle L, Zacharaki EI, Robert C, Reuzé S, Schernberg A, Paragios N, Deutsch E and Ferté C: Promises and challenges for the implementation of computational medical imaging (radiomics) in oncology. Ann Oncol 28: 1191-1206, 2017.

32. Li Y, Liu X, Xu K, Qian Z, Wang K, Fan X, Li S, Wang Y and Jiang T: MRI features can predict EGFR expression in lower grade gliomas: A voxel-based radiomic analysis. Eur Radiol 28: 356-362, 2018.

33. Li Z, Sun J, Chen L, Huang N, Hu P, Hu X, Han G, Zhou Y, Bai W, Niu T and Yang X: Assessment of liver fibrosis using pharmacokinetic parameters of dynamic contrast-enhanced magnetic resonance imaging. Magn Reson Imaging 44: 98-104, 2016.

34. Shan QY, Hu HT, Feng ST, Peng ZP, Chen SL, Zhou Q, Li X, Xie XY, Lu MD, Wang W and Kuang M: CT-based peritumoral radiomics signatures to predict early recurrence in hepatocellular carcinoma after curative tumor resection or ablation. Cancer Imaging 19: 11, 2019.

35. Khalifa F, Soliman A, El-Baz A, Abou El-Ghar M, El-Diasty T, Gimel'farb G, Ouseph R and Dwyer AC: Models and methods for analyzing DCE-MRI: A review. Med Phys 41: 124301, 2014.

36. Rizzo S, Botta F, Raimondi S, Origgi D, Fanciullo C, Morganti AG and Bellomi M: Radiomics: The facts and the challenges of image analysis. Eur Radiol Exp 2: 36, 2018.

This work is licensed under a Creative Commons Attribution-NonCommercial-NoDerivatives 4.0 International (CC BY-NC-ND 4.0) License. 